'Unidad de Hematología Clínica, Sección de Hematología, Hospital del Salvador. Santiago, Chile. 2Sección de Hematología.

Departamento de Medicina Oriente, Facultad de Medicina. Universidad de Chile. Santiago, Chile.

aResidentes de Hematología, Hospital del Salvador, Santiago. Universidad de Chile. Santiago, Chile.

Trabajo no recibió financiamiento. Los autores declaran no tener conflictos de interés.

Recibido el 7 de enero de 2019, aceptado el 23 de mayo de 2019.

Correspondencia a: Dra. Camila Peña Av Salvador 364, Providencia. Santiago, Chile. camipena@gmail.com

\section{Anemia hemolítica autoinmune en Chile: un análisis retrospectivo de 43 pacientes}

\author{
HERNÁN LÓPEZ-VIDAL ${ }^{\mathrm{a}}$, CAMILA PEÑA ${ }^{1}$, CLAUDIA GAJARDO $^{\mathrm{a}}$, \\ XIMENA VALLADARES ${ }^{1}$, MARÍA ELENA CABRERA C. ${ }^{2}$
}

\section{Autoimmune hemolytic anemia. Review of 43 cases}

Background: Autoimmune hemolytic anemia (AIHA) is an uncommon disease. In its presentation, it can be severe and even lethal. There is only one clinical report concerning this pathology in Chile. Objective: To describe the clinical characteristics and evolution of adult AIHA inpatients. Materials and Methods: Retrospective review of clinical records of adult AIHA inpatients between January 2010 and June 2018 was done. Demographic, clinical, laboratory and therapeutic information was analyzed. A descriptive, analytical and survival analysis was performed. Results: Forty-three adult patients diagnosed with AHIA were hospitalized in a period of 8 years. Median age was 63 years (range 22-86 years), mostly women (72\%). Warm antibodies were detected in 36 cases (84\%) and cold antibodies in seven. Seventy two percent of the patients had an underlying cause, and 58\% were secondary to lymphoproliferative neoplasms. All patients except two, received steroids as initial treatment, with response in 37 (90\%) of them. Three refractory patients received rituximab, with response in all of them, and relapse in one. Median follow-up was 38 months (range 2-98 months). Five year overall survival was $72 \%$. Conclusion: AHIA in adults inpatients is a heterogeneous disease, mainly due to warm antibodies, and to secondary etiology.

(Rev Med Chile 2019; 147: 836-841)

Key words: Autoimmune Diseases; Autoantibodies; Anemia, Hemolytic, Autoimmune; Rituximab.
L a anemia hemolítica autoinmune (AHAI) es una enfermedad infrecuente y heterogénea en su presentación, causada por anticuerpos (Ig) dirigidos contra antígenos (Ag) de la superficie de los glóbulos rojos. Se estima una incidencia de 1-3/100.000 habitantes por año ${ }^{1}$. Se clasifican de acuerdo a las propiedades térmicas de los Ig, AHAI por Ig calientes en $80-90 \%$, Ig fríos y mixtos en el resto ${ }^{1,4}$. La AHAI puede ser subdividida en primaria (o idiopática) y secundaria, dependiendo de la presencia o no de una causa asociada ${ }^{3}$.
Aproximadamente en la mitad de los casos existe una causa conocida, siendo las principales los síndromes linfoproliferativos y las mesenquimopatías (50 y $40 \%$, respectivamente). El resto se compone de causas infecciosa, fármacos u otras ${ }^{1,4}$.

La presentación clínica es heterogénea, desde formas leves a muy graves, con tasas de mortalidad descritas de hasta $11 \%$ en series antiguas y de aproximadamente $4 \%$ en estudios retrospectivos recientemente publicados.

En la literatura nacional encontramos solo un 
reporte de un caso clínico de AHAI asociada a virus de inmunodeficiencia humana ${ }^{5}$.

Nos pareció de interés analizar de forma retrospectiva las características clínicas y evolución de pacientes con el diagnóstico de AHAI hospitalizados en nuestra institución.

\section{Materiales y Métodos}

\section{Pacientes}

Se evaluaron los pacientes con diagnóstico de AHAI hospitalizados y tratados por la Sección de Hematología del Hospital del Salvador de Santiago, entre enero de 2010 y junio de 2018. El estudio de laboratorio analizado fue el siguiente: hemograma, porcentaje de reticulocitos, perfil bioquímico, deshidrogenasa láctica (LDH), bilirrubina directa e indirecta, test de Coombs directo e indirecto, crioaglutininas (ante sospecha clínica), anticuerpos antinucleares, factor reumatoideo, complemento C3 y C4, serología para virus de la inmunodeficiencia humana, virus hepatitis B y C. En pacientes en los que se sospechó un síndrome linfoproliferativo se realizó estudio de médula ósea y tomografía axial computarizada.

Se diagnosticó un síndrome de Evans si además de la AHAI se presentaba una trombocitopenia o neutropenia inmune.

\section{Tratamiento}

El tratamiento estándar de primera línea fue prednisona oral $1 \mathrm{mg} / \mathrm{kg} /$ día por al menos 3 semanas, dexametasona $40 \mathrm{mg}$ intravenosa (iv) por 4 días, o metilprednisolona iv $500 \mathrm{mg} /$ día por 3 días. Como segunda línea se utilizó tratamiento con rituximab o esplenectomía. Azatioprina y ciclofosfamida oral se utilizó en pacientes refractarios. Entre los años 2015 y 2018, se utilizó rituximab como primera línea, a dosis de $375 \mathrm{mg} / \mathrm{m}^{2} /$ semana por 4 semanas consecutivas, en casos de AHAI por Ig fríos. Antes se usó corticoides.

El criterio de respuesta fue: a) remisión completa ( $\mathrm{RC})$ : mantener hemoglobina $(\mathrm{Hb})$ estable mayor o igual a $12 \mathrm{~g} / \mathrm{dl}$, sin signos de hemólisis (reticulocitos, bilirrubina y LDH normales) y sin necesidad de tratamiento; b) remisión parcial (RP): Hb mayor o igual a $12 \mathrm{~g} / \mathrm{dl}$ y necesidad de mantener corticoides y c) no respuesta: necesidad de asociar otra terapia a los corticoides. Se consideró buena respuesta o respuesta óptima a pacientes que lograron RC o RP.

\section{Análisis estadísticos}

Se realizó estadística descriptiva y estadística analítica con test $\mathrm{t}$-Student, prueba exacta de Fisher o chi cuadrado, según fuera el caso. Se analizó la sobrevida con curva de Kaplan y Meier y se realizó comparación con test de log rank. Se consideró significativo un $\mathrm{p}<0,05$. Se utilizó el programa STATA 12.

Este estudio contó con la aprobación del Comité de Ética del establecimiento.

\section{Resultados}

\section{Características de los pacientes}

Se hospitalizaron 43 pacientes con diagnóstico de AHAI en el período señalado de 8 años. La edad media fue 63 años (rango 22-86 años) y con predominio de mujeres (72\%). Entre las comorbilidades se objetivaron enfermedades autoinmunes en 12 casos, destacando el hipotiroidismo, vitíligo, síndrome de Sjögren y anemia perniciosa. Las características clínicas y de laboratorio se observan en la Tabla 1.

El test de Coombs directo fue negativo solo en un caso $(2,5 \%)$. En 10 pacientes $(23 \%)$ se observó asociación con trombocitopenia inmune (síndrome de Evans). En 31 pacientes (72\%) se detectó una causa subyacente (formas secundarias). De estos, 18 casos (58\%) (Figura 1) correspondieron a un síndrome linfoproliferativo (8 leucemias linfáticas crónicas [LLC], 3 linfomas no Hodgkin [LNH] marginales esplénicos, 2 LNH linfoplasmocíticos, 2 LNH T angioinmunoblástico, 2 linfomas de Hodgkin y 1 caso de linfocitosis B monoclonal), 7 casos $(23 \%)$ fueron secundarios a mesenquimopatía ( 3 lupus eritematoso sistémico, 2 síndromes antifosfolípidos, y 1 caso de artritis reumatoide y 1 de dermatomiositis). Se objetivó un tumor sólido asociado en 3 casos ( 2 gástricos y 1 de mama) y un síndrome mielodisplástico en 2 casos. Un caso de AHAI secundaria fue asociado a infección por Mycoplasma pneumoniae. Las características de los pacientes con AHAI secundaria se muestran en la Tabla 2.

En 30 casos, el diagnóstico de AHAI fue concomitante al diagnóstico de la enfermedad de base y en uno se realizó con posterioridad. En todos los pacientes con AHAI por Ig fríos se detectó una etiología de base. No se registró casos de causa secundaria a drogas.

La edad media de los pacientes con AHAI 
Tabla 1. Características clínicas y de laboratorio de pacientes con AHAl $(p<0,05)$

\begin{tabular}{|lcccc|}
\hline & Total & Primaria & Secundaria & p-value \\
\hline Total, $n(\%)$ & $43(100)$ & $12(28)$ & $31(72)$ & NS \\
\hline Mujer, (\%) & 72 & 83 & 71 & NS \\
\hline Edad, media (rango) & $63(22-86)$ & $50(22-81)$ & $63(27-86)$ & NS \\
\hline Sindrome de Evans, $n(\%)$ & $10(23)$ & $3(27)$ & $7(22)$ & NS \\
\hline Hb media, g/dl & $5,9( \pm 1,26)$ & $6,2( \pm 0,86)$ & $5,8( \pm 1,33)$ & NS \\
LDH media, U/L & $650( \pm 700)$ & $902( \pm 974)$ & $541( \pm 564)$ & NS \\
\hline LDH elevada, \% & 72 & 80 & 68 & NS \\
\hline Bilirrubina total, mg/dl & $2,8( \pm 2,5)$ & $2,01( \pm 1,31)$ & $3,1( \pm 2,93)$ & NS \\
\hline Bilirrubina total elevada, \% & 64 & 64 & 56 & \\
\hline
\end{tabular}

NS: no estadísticamente significativo. $\mathrm{Hb}$ : hemoglobinemia. LDH: deshidrogenasa láctica.

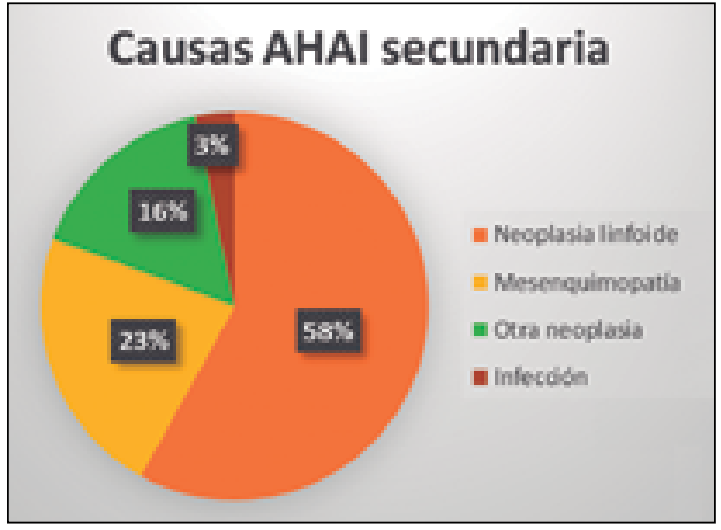

Figura 1. Muestra los porcentajes de las causas encontradas en el presente estudio. por Ig calientes fue 55 años, en cambio la de los pacientes con Ig fríos fue 67 años. La anemia fue significativamente mayor en los pacientes con AHAI por Ig calientes. Las características de los pacientes según el tipo de anticuerpo se muestra en la Tabla 3.

\section{Respuesta al tratamiento}

Todos los pacientes con AHAI por Ig calientes recibieron corticoides como tratamiento inicial, además del tratamiento de la causa subyacente en los casos secundarios. En $92 \%$ de estos casos (33/36) se logró respuesta óptima. Dos respondieron a rituximab como segunda línea y 1 caso a ciclofosfamida. Cinco pacientes $(14 \%)$ recayeron,

Tabla 2. Características de los pacientes con AHAl secundaria

\begin{tabular}{|lcccc|}
\hline & Neoplasia linfoide & Mesenquimopatía & Otra neoplasia & Infección \\
\hline Pacientes, $n$ & 18 & 7 & 5 & 1 \\
Hombre/Mujer, n (\%) & $7(39) / 11(61)$ & $0(0) / 7(100)$ & $2(33) / 3(66)$ & $0(0) / 1(100)$ \\
\hline Edad media, (rango) & $63(27-86)$ & $53(29-74)$ & $71(38-81)$ & 66 \\
Síndrome de Evans, n (\%) & $4(22)$ & $2(29)$ & $1(33)$ & $0(0)$ \\
Hb media, g/dl & 5,4 & 7,1 & 5,1 & 8 \\
LDH media, U/L & 359 & 445 & 942 & 2150 \\
\hline Bilirrubina, mg/dl & 2,06 & 4,7 & 4,4 & 6,4 \\
\hline Ac. Calientes/Fríos, n (\%) & $14(78) / 4(22)$ & $6(85) / 1(13)$ & $4(80) / 1(20)$ & $0(0) / 1(100)$ \\
\hline
\end{tabular}

$\mathrm{Hb}$ : hemoglobinemia. LDH: deshidrogenasa láctica. 
Tabla 3. Características de los pacientes con AHAl según el tipo de anticuerpo $(p<0,05)$

\begin{tabular}{|lccc|}
\hline & Ig calientes & Ig fríos & p-value \\
\hline Total, $\mathrm{n}(\%)$ & $36(84)$ & $7(16)$ & 1 \\
\hline Hombre/Mujer, $\mathrm{n}(\%)$ & $10(28) / 26(72)$ & $2(29) / 5(71)$ & 0,09 \\
\hline Edad media, (rango) & $55(22-81)$ & $67(58-79)$ & 0,31 \\
Síndrome de Evans, $\mathrm{n}(\%)$ & $9(25)$ & $0(0 \%)$ & 0,003 \\
Hb, g/dl & 5,8 & 6,4 & 0,35 \\
LDH media, U/L & 449 & 794 & 0,48 \\
\hline Bilirrubina media, mg/dl & 2 & 3,8 & 0,41 \\
\hline Respuesta a corticoides, $\mathrm{n}(\%)$ & $33 / 36(92)$ & $4 / 5(80)$ & \\
\hline
\end{tabular}

Hb: hemoglobinemia. LDH: deshidrogenasa láctica.

1 respondió posteriormente a azatioprina, y 4 a un nuevo ciclo de corticoides. Cinco de 7 pacientes con AHAI por Ig fríos recibieron tratamiento según la etiología subyacente además de corticoides. Hubo respuesta en $80 \%$ de los casos ( 4 de 5). Dos casos se trataron con rituximab como primera línea, logrando respuesta óptima. Un paciente fue refractario a corticoides, respondiendo posteriormente a rituximab en segunda línea.

El seguimiento medio fue 38 meses (rango 2-98 meses). Nueve pacientes han fallecido, ninguno por la anemia propiamente tal, sino por la causa subyacente: 3 casos por cáncer gástrico, 3 por LLC, y uno por LNH. La sobrevida global a 5 años fue $72 \%$ (Figura 2).

\section{Discusión}

El presente estudio concuerda con lo publicado en cuanto a que la mayoría de las AHAI son mediadas por Ig calientes, cifra que oscila entre 80 y $90 \%$, y predomina en mujeres. La AHAI por Ig fríos ocurre a mayor edad, lo que confirmamos en nuestra revisión.

Creemos importante destacar que la hiperbilirrubinemia indirecta y el aumento de LDH, criterios característicos clásicos de hemólisis, no fueron observados en aproximadamente un tercio de los casos. Otro examen relevante para el diagnóstico de AHAI es el test de Coombs. En nuestra revisión, el test de Coombs directo fue positivo en todos los casos, excepto en uno. Esta paciente presentó un síndrome de Evans, claros

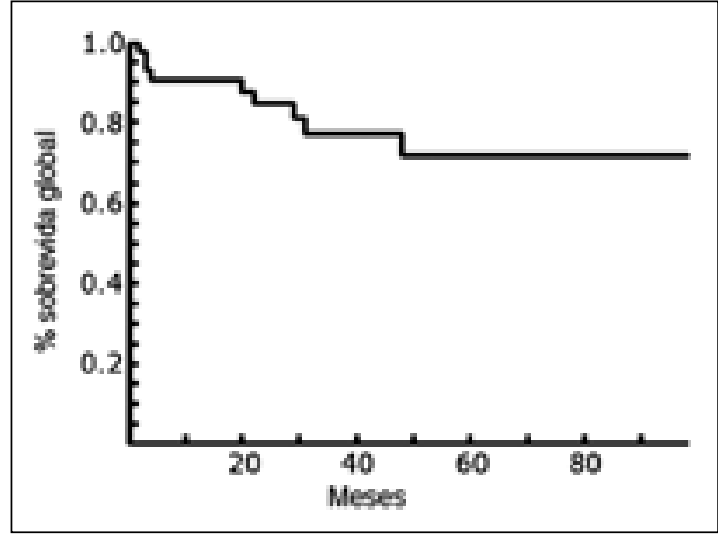

Figura 2. Sobrevida global de los 43 pacientes con AHAl.

signos de hemolisis de laboratorio y era portadora de un carcinoma gástrico. La literatura describe que entre $2 \%$ y $10 \%$ de los pacientes tienen un test de Coombs negativo ${ }^{1,6}$ dependiendo de la sensibilidad del reactivo.

Clásicamente se describe que aproximadamente la mitad de los casos son secundarios. En nuestra revisión esta asociación fue muy superior: $72 \%$ de los casos demostró una causa subyacente, tendencia que ha sido evidenciada en recientes publicaciones de series de $\operatorname{casos}^{3,16,17}$. La mayor parte fueron síndromes linfoproliferativos. En nuestra cohorte, esto puede explicarse por la mayor sospecha y capacidad diagnóstica de neoplasia hematológicas al ser nuestro hospital un centro de referencia nacional en hematología. 
En un solo paciente se diagnosticó un síndrome linfoproliferativo posterior a el debut de la AHAI, cifra menor a lo publicado. Sallah ${ }^{7}$, en su estudio de 107 casos con AHAI, mostró que 18\% desarrolló una neoplasia linfoproliferativa, con una media de 26 meses después del debut de la anemia hemolítica.

La AHAI por Ig fríos constituyó 16\% del total de casos, cifra inferior a la de otros países, por ejemplo, en Noruega constituye hasta $25 \%{ }^{8,9}$. El estudio GIMEMA ${ }^{6}$ reporta 308 pacientes con AHAI primaria, en los que la anemia por Ig fríos correspondió a 27\% del total de casos. Esta menor incidencia puede deberse a un subdiagnóstico en nuestro centro.

Todos los pacientes con AHAI por Ig fríos tuvieron una causa, lo que es consistente con lo publicado, siendo el linfoma linfoplasmocítico el más frecuente ${ }^{9}$.

Destacamos, además, que la anemia fue significativamente más intensa que en los pacientes con AHAI por Ig calientes.

Respecto al tratamiento, hay consenso que la terapia de primera línea son los corticoides ${ }^{10-13}$, especialmente en AHAI por Ig calientes. Nuestra revisión evidencia cifras de respuesta comparables a las publicadas, las que describen una tasa de respuesta de alrededor de $80 \%$.

A pesar de que la esplenectomía se describe clásicamente como una segunda línea de tratamiento, no se realizó en ninguno de nuestros pacientes. Esto se condice con la tendencia mundial de evitar este procedimiento. Por lo demás, se ha comprobado que no tiene mayor efectividad en AHAI secundaria, grupo en el cual se encuentran la mayoría de nuestros pacientes ${ }^{1-4}$.

El rituximab se recomienda en segunda línea con respuesta global de $40 \%$ a $80 \%$. Todos nuestros pacientes respondieron, con un tercio de recaídas, lo que coincide con lo descrito en la literatura.

La respuesta al tratamiento inmunosupresor con ciclofosfamida/azathioprina fue de $66 \%$, similar a lo publicado, que varía entre $60 \%$ y $84 \%{ }^{10,14}$.

La respuesta a los corticoides en la AHAI por Ig fríos es menor, lo que se reproduce en nuestros pacientes. La recomendación en primera línea en este tipo de AHAI es el rituximab, ya que actúa directamente contra el linfocito $\mathrm{B}$ clonal. Las respuestas varían entre $45 \%$ y $58 \%$, siendo mayor en nuestra cohorte. Sin embargo, el número de pacientes con AHAI de Ig fríos en nuestra serie es reducido, lo que podría explicar esta diferencia ${ }^{12,13}$.

La sobrevida global fue menor a lo reportado en otras series, las que la describen por sobre $80 \%$ a 5 años ${ }^{15}$. Una explicación podría ser el mayor número casos de AHAI secundaria, especialmente a neoplasias hematológicas y tumores sólidos, ya que la mayoría de los fallecidos fue por neoplasia.

En nuestro centro, los pacientes con AHAI grave que requieren hospitalización, tienen características similares a lo descrito en la literatura. Sin embargo, nuestra revisión de casos mostró a las AHAI secundarias como las más frecuentes, lo que pudo determinar la mayor mortalidad observada. Por este motivo creemos que es necesario el estudio exhaustivo dirigido a descartar una etiología subyacente en todo paciente en el que se diagnostique con AHAI.

\section{Referencias}

1. Bass GF, Tuscano MT, Tuscano JM. Diagnosis and classification of autoimmune hemolytic anemia. Autoimmun Rev 2014; 13: 560-4.

2. Seve P, Philippe P, Dufoir JP, Broussolle C, Michel M. Autoimmune hemolytic anemia: classification and therapeutic approaches. Expert Rev Hematol 2008; 189-204.

3. Roumier M, Valentine Loustau V, Guillaud C, Languille L, MahevasM, Khellaf M, et al. Characteristics and outcome of warm autoimmune hemolytic anemia in adults: new insights based on a single-center experience with 60 patients. Am J Hematol 2014; 89: E150-5.

4. Barcellini W, Fattizzo B. Clinical Applications of Hemolytic Markers in the Differential Diagnosis and Management of Hemolytic Anemia. Dis Markers 2015; 2015: 635670.

5. Enberg M, Yaquich P, Pilasi C, Thompson L, Oddó D, Bronfman L. Anemia hemolítica autoinmune en paciente infectado por virus de inmunodeficiencia humana $y$ enfermedad de Castleman. Caso clínico. Rev Chilena Infectol 2002; 19 (4): 231-6.

6. Barcellini W, Fattizzo B, Zaninoni A, Radice T, Nichele I, Di Bona E, et al. Clinical heterogeneity and predictors of outcome in primary autoimmune hemolytic anemia: a GIMEMA study of 308 patients. Blood 2014; 124 (19): 2930-6.

7. Sallah S, Wan JY, Hanrahan LR. Future development of lymphoproliferative disorders in patients with autoimmune hemolytic anemia. Clin Cancer Res 2001; 7: $791-4$. 
8. Berentsen S, Randen U, Tjønnfjord GE. Cold Agglutinin-Mediated Autoimmune Hemolytic Anemia. Hematol Oncol Clin N Am 2015; 29: 455-71.

9. Berentsen S, Ulvestad E, Langholm R, Beiske K, Hjorth-Hansen H, Ghanima W, et al. Primary chronic cold agglutinin disease: a population based clinical study of 86 patients. Haematologica 2006; 91(4): 460-6.

10. Liebman HA, Weitz IC. Autoimmune Hemolytic Anemia. Med Clin N Am 2017; 101: 351-9.

11. Maung SW, Leahy M, O'Leary HM, Khan I, Cahill MR, Gilligan $\mathrm{O}$, et al. A multi-centre retrospective study of rituximab use in the treatment of relapsed or resistant warm autoimmune haemolyticanaemia. Br J Haematol 2013; 163: 118-22.

12. Schollkopf C, Kjeldsen L, Bjerrum OW, Mourits-Andersen HT, Nielsen JL, Christensen BE, et al. Rituximab in chronic cold agglutinin disease: a prospective study of 20 patients. Leuk Lymphoma 2006; 47 (2): 253-60.
13. Go RS, Winters JL, Kay NE. How I treat autoimmune hemolytic anemia. Blood 2017; 129: 2971-9.

14. Michel M. Warm autoimmune hemolytic anemia: Advances in pathophysiology and treatment. Presse Med 2014; 43: e97-e104. 12.

15. Rattarittamrong E, Eiamprapai P, Tantiworawit A, Rattanathammethee T, Hantrakool S, Chai-Adisaksopha C, et al. Clinical characteristics and long-term outcomes of warm-type autoimmune hemolytic anemia. Hematology 2016; 21 (6): 368-74.

16. Baek SW, Lee MW, Ryu HW, Lee KS, Song IC, Lee HJ, et al. Clinical features and outcomes of autoinmune hemolytic anemia: a retrospective analysis of 32 cases. Korean J Hematol 2011; 46 (2): 111-7.

17. Sudulagunta SR, Kumbhat M, Sodalagunta MB, Nataraju AS, Bangalore Raja SK, Thejaswi KC, et al. Warm Autoimmune Hemolytic Anemia: Clinical Profile and Management. J Hematol 2017; 6 (1): 12-20. 